\title{
Real-time and spatially resolved assessment of pathogens in crops for site-specific pesticide reduction strategies
}

\author{
V. Kilin ${ }^{1}$, V. Pini ${ }^{1}$, J. Kasparian ${ }^{1,2}$, S. Gros ${ }^{3}$, and J.-P. Wolf ${ }^{1, \text { a }}$ \\ ${ }^{1}$ Dept. of Applied Physics, Univ. Geneva, 1211 Genève 4, Switzerland \\ ${ }^{2}$ Inst. for Environmental Sciences, Univ. Geneva, 1211 Genève 4, Switzerland \\ ${ }^{3}$ Cave Stéphane Gros, Chemin de Bertholier 10, 1283 Dardagny/GE, Switzerland
}

\begin{abstract}
We deploy a network of autonomous stations measuring meteorological and soil parameters, as well as the airborne particle size distribution with a focus on the size of Plasmapora Viticola (PV). They provide early warning and detection of PV spore outbursts with high spatial and temporal resolution. We evidence the high spatial inhomogeneity of this pathogen, potentially allowing to limit treatments to the specific times and locations where infection risk is detected.
\end{abstract}

\section{Introduction}

The use of pesticides in agriculture has many drawbacks, with impacts on product costs, product quality, soil and air pollution, as well as human health [1]. Significant efforts are dedicated to reducing their use, including governmental regulation strategies. Several options for pesticide reduction are considered in this respect, including genetically modified organisms (GMO), development of more "environmental friendly" pesticides, and technologies yielding more targeted treatments.

One strategy in this respect is to more selectively treat plantations in time and space, with the assistance of smart sensing and prediction models. For monitoring of plant disease spread one can mention 4 recent developments: (1) highly sensitive and selective devices, which detect pathogens in the air; (2) sampling of leaves and further analysis in the laboratory; (3) modeling using meteorological data and their link to the parasite's biology and development; and (4) hyperspectral imaging of the fields from drones or satellites.

A major issue for (1), (2) and (3) is the spatial representativeness of the assessments over the entire production field, because data are usually only representative locally in space and/or time. Predictive modeling using meteorological data (air temperature and humidity, precipitation values, wind) suffer from the same spatial sparsity, and thus can't account for local conditions like topography, proximity to a river or a forest, soil quality, shadowing, turbulence, local and/or chemical treatment history, etc. Moreover, they don't assess the presence of the sources of pathogens, nor the further spread of spores. Airborne (e.g. drone based) hyperspectral cameras, on the other hand, provide large scale assessments with high resolution in space (however not in time), but they detect sickness or stress of the foliage once it already occurred, lacking a satisfactory predictive information for the producer.

a e-mail: jean-pierre.wolf@unige.ch
In this context, a systematic study of the spatial and temporal heterogeneity of the infection agents is highly valuable [2]. In the present study, we address this issue by deploying a network of autonomous stations over a field, which measures airborne spores, environmental conditions, and soil parameters in real time. A possible, but not exclusive, way of using this large data set is to apply Artificial intelligence (AI) to identify patterns representative to the infection risk. The long term objective of the work is to provide tools to the producers for accurately and selectively treat vineyards only where and when treatment is needed rather than over the whole field on a periodic basis, adapted to rain washing events.

\section{Measurement stations and network}

Our first experimental assessment in 2018 (June to October) was focused on the prevention of Downy Mildew infection. We deployed a network of 5 autonomous (solar powered) stations over vineyards of ca. $50 \mathrm{ha}$, with an average distance between neighboring stations of ca. $400 \mathrm{~m}$. Each station provided the following realtime information: concentration and size distribution of airborne particles, i.e. Plasmopara Viticola (PV) spores, temperature and relative humidity $(\mathrm{RH})$ of the air, temperature and humidity of the soil, leaf humidity and solar irradiance. The stations were inter-compared and calibrated with reference devices (Grimm 1.109, Grimm 1.365, Testo 635) prior to field deployment. The acquired data were centralized on a server and processed by AI. The fitness function for the algorithm was obtained by weekly-integrated impaction filters, and analysed in the laboratory by multimodal, multiphoton microscopy $[3,4]$. The latter method, providing 3D images of the spores at highest spatial resolution, as well as spectrally resolved (32 channels) multi-photon excited fluorescence proved very reliable for identifying PV spores on the filters.

Significant weekly variation (up to a factor of 10) in PV spore concentrations are observed in our measurements over the 7 weeks shown in Fig. 1. As compared 


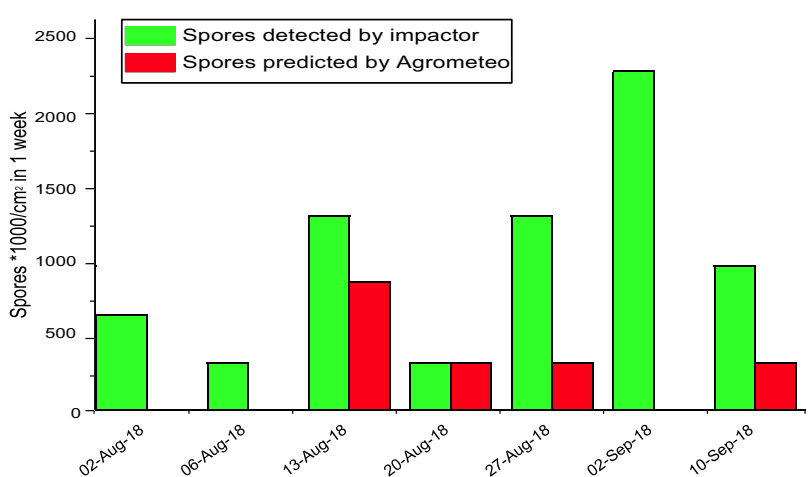

Figure 1. Local counting of Plasmopara Viticola spores using weekly averaged sampling on filters and multimodal microscopy identification. Also shown: forecasts from meteorology based modeling (Agrometeo, Agroscope, Changins, Switzerland).

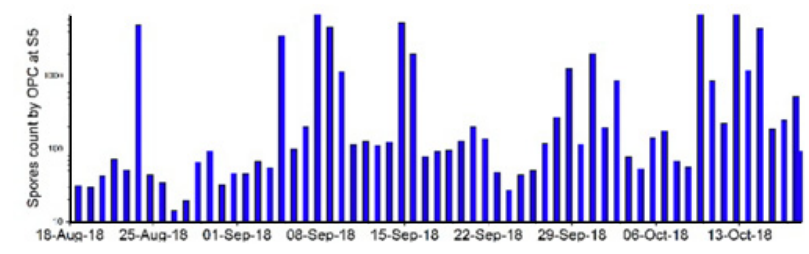

Figure 2. Daily variation of airborne particles acquired by the laser OPC in the size bins of PV (spheroids 12-16 um) during 2 months at the location of station 5 . Notice the vertical logarithmic scale.

to the predictions from the meteorological model, the concentrations lie in the same range, but differences between the most and the least exposed weeks are observed. This is attributed to several parameters, such as the local character of the spore sources, the effect of chemical treatments in the field, wind and turbulence, as well as the specific topology of the sampling station location, which is close to a stream (S5 in Fig. 3). The spatial heterogeneity over the field is detailed in the next section on the basis of the measurements from the 5 laser- based real-time optical particle counters (OPC). Also, as mentioned in the introduction, direct quantitative measurements of the PV spores like the ones presented in Fig. 1 were used as fitness function for the AI handling of the network stations data.

\section{Spatial and temporal heterogeneity}

Although the full data set (size resolved concentrations of spores, temperature and humidity in the soil and in the air, solar irradiance, etc.) was recorded and analysed, we concentrate now on the measurements of airborne particles.

As an example, Fig. 2 shows the temporal variation of airborne particle concentration in the size bins corresponding to the spores with size comparable to PV for one specific station. Real-time (every $3 \mathrm{~s}$ ) measurements are aggregated into daily-averaged values. On this time scale, variations span over more than 2 orders of magnitude on this specific location. Interestingly, steadily increasing concentrations over a 5 to 8 days are observed. Such dynamics in spore's concentration are in good agreement with well characterized durations of sporulation's periods [5]. These high concentrations, in turn, can be crosscorrelated to meteorological conditions. The correlations
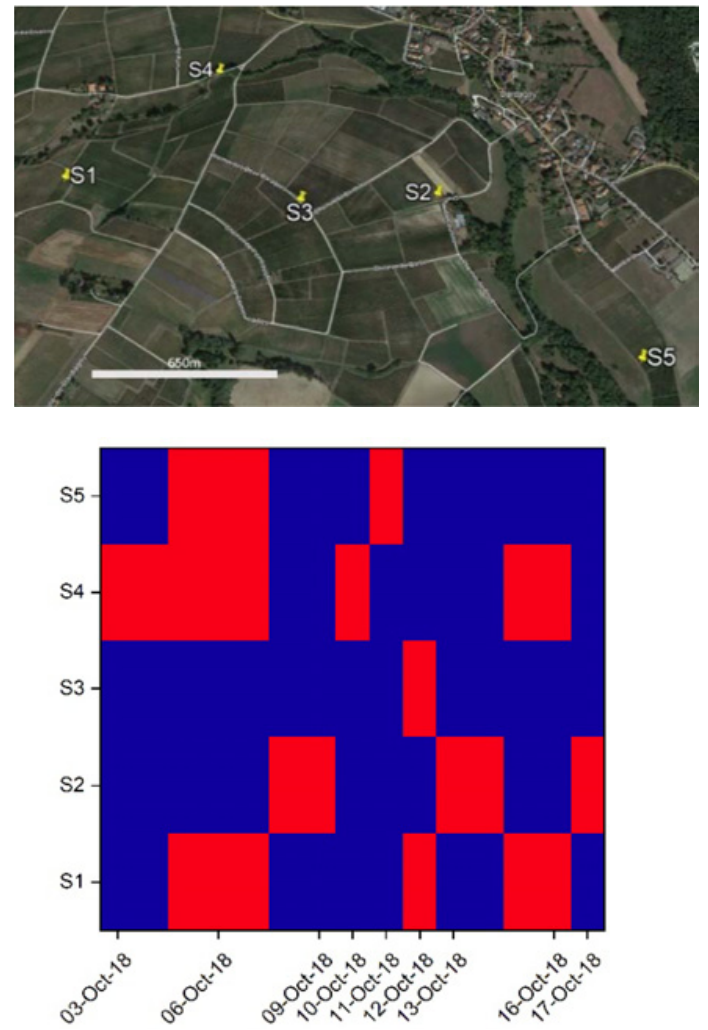

Figure 3. Comparison of the high-particle concentration events (daily averaged - red rectangles) between the 5 measurement sites, summarizing spatio-temporal heterogeneity over the field. Shown period: Oct. 3-Oct 17, 2018.

between temperature/humidity and spore concentration indicate that predicting the number of airborne spores using these proxys is difficult. Similar conclusions were recently reported by Carisse et al. [6,7].

As mentioned, five real-time measuring stations were deployed, so that inter-comparisons between different sites could be performed (Fig. 3). Sites were specifically selected to exhibit different characteristics in term of solar radiation/shadowing, wind exposure, proximity to water, etc. As an illustrative example, Fig. 3 displays the occurrence of high spore concentration events as a function of time (daily averaged values), for the 5 stations. No systematic time correlation is observed, although some patterns seem to be delayed by 1-2 days between the stations, the origin of which could be attributed to spore transport. Also, the total number of events differs significantly from one site to another, as for example the low numbers observed for S3 in shown timeframe. These results demonstrate strong heterogeneity of spore's evolution in fields at sub kilometer scale and open new possibilities for the reduction of pesticides.

Significant efforts have been dedicated in the literature to the assessment of the spore concentration inhomogeneity in vineyards, but none were really conclusive. The main issue is the simultaneous availability of spatially resolved data and high temporal resolution. For instance, many studies used impactors, traps, filters or leaves, but with temporal resolutions of the order of the week. Conversely, a drone based detector can exhibit fast sampling rates, but cannot measure simultaneously at different locations and induces wind turbulence affecting 
the measurement accuracy. In this respect, our approach of deploying networks of cost-effective stations constitutes a valuable option.

Former studies [9] reported that by combining environment monitoring and airborne spores concentrations measurements, $40 \%$ fewer fungicides spraying could be used as compared to calendar-based schedule spraying. The spore detection, however, was based on the use of traps and consecutive laboratory assessment, which appears difficult to generalize as a standard procedure. In this respect also, our approach of deploying networks of realtime OPCs constitutes therefore a valuable option [10].

The reported measurement campaign in 2018 corresponds only to a first step in our efforts towards a real time assessment of the contamination risk, based on the direct, spatially resolved, detection of pathogens. A combination with high level modeling like Agrometeo (Agroscope, Changins, Switzerland), which takes into account the biological development of the pathogens and its relation to meteorological and soil parameters appears, furthermore, a very attractive combination for reducing the number of pesticide treatments per season in a targeted and safe manner.

First of all, we wish to sincerely thank the "Vignerons, Viticulteurs et Encaveurs" of Dardagny for their great collaboration, participation and support during this campaign, in particular Emilienne Hutin.
The authors acknowledge funding from the NCCR MUST, Swiss NSF and the Geneva Creativity Center (GCC, https://creativitycenter.ch).

\section{References}

[1] M. Lechenet, F. Dessaint, G. Py, D. Makowski, N. Munier-Jolain, Nat. Plants 3, 6 (2017)

[2] M.L. Fall, H. Van der Heyden, L. Brodeur, Y. Leclerc, G. Moreau, O. Carisse, Plant Pathol. 64, 178 (2015)

[3] S. Bellow, G. Latouche, S.C. Brown, A. Poutaraud, Z.G. Cerovic, J. Exp. Bot. 63, 3697 (2012)

[4] V. Kilin, C. Mas, S. Constant, J.P. Wolf, L. Bonacina. Sci. Rep. 7, 16233 (2017)

[5] T. Caffi, G. Gilardi, M. Monchiero, V. Rossi, Phytopathol. 103, 64 (2013)

[6] O. Carisse, H. van der Heyden, V. MorissetteThomas, ActaHortic. 1068, 179 (2015)

[7] X.J. Kong, W.T. Qin, X.Q. Huang, F.F. Kong, C.D. Schoen, J. Feng, Z.Y. Wang, H. Zhang, Sci. Rep. 6, 9 (2016)

[8] W.F. Mahaffee, R. Stoll, Phytopathol. 106, 420 (2016)

[9] O. Carisse, R. Bacon, A. Lefebvre, Crop Prot. 28, 1036 (2009)

[10] V. Kilin, J. Kasparian, J.P. Wolf, Patent EP19170073 (2019) pending 